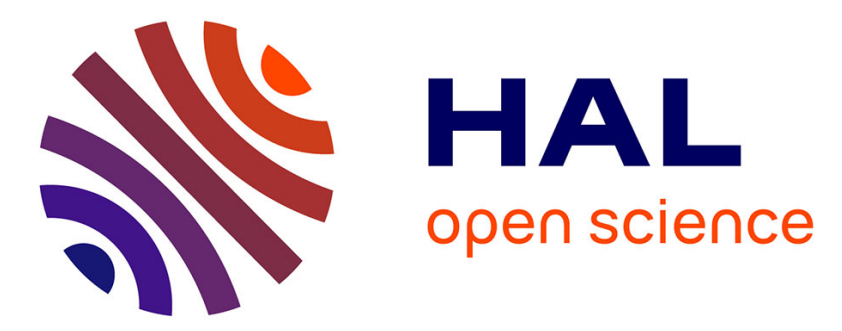

\title{
Ab Initio Nonequilibrium Thermodynamic and Transport Properties of Ultrafast Laser Irradiated 316L Stainless Steel
}

Emile Bévillon, Jean-Philippe Colombier, Biswanath Dutta, Razvan Stoian

\section{- To cite this version:}

Emile Bévillon, Jean-Philippe Colombier, Biswanath Dutta, Razvan Stoian. Ab Initio Nonequilibrium Thermodynamic and Transport Properties of Ultrafast Laser Irradiated 316L Stainless Steel. Journal of Physical Chemistry C, 2015, 119 (21), pp.11438-11446. 10.1021/acs.jpcc.5b02085 • ujm-01159667

\section{HAL Id: ujm-01159667}

\section{https://hal-ujm.archives-ouvertes.fr/ujm-01159667}

Submitted on 5 Jun 2015

HAL is a multi-disciplinary open access archive for the deposit and dissemination of scientific research documents, whether they are published or not. The documents may come from teaching and research institutions in France or abroad, or from public or private research centers.
L'archive ouverte pluridisciplinaire HAL, est destinée au dépôt et à la diffusion de documents scientifiques de niveau recherche, publiés ou non, émanant des établissements d'enseignement et de recherche français ou étrangers, des laboratoires publics ou privés. 


\title{
Ab Initio Nonequilibrium Thermodynamic and Transport Properties of Ultrafast Laser Irradiated 316L Stainless Steel
}

\author{
E. Bévillon, ${ }^{\dagger}$ J.P. Colombier, ${ }^{*} \dagger$ B. Dutta, ${ }^{\ddagger}$ and R. Stoian $^{\dagger}$ \\ †Laboratoire Hubert Curien, UMR CNRS 5516, Université de Lyon, \\ Université Jean-Monnet 42000, Saint-Etienne, France \\ †Max-Planck-Institut für Eisenforschung GmbH, D-40237, Düsseldorf, Germany \\ E-mail: jean.philippe.colombier@univ-st-etienne.fr \\ Phone: +334779158 82
}

\section{Abstract}

We present calculations of transient behavior of thermodynamic and transport coefficients on the timescale of electron-phonon relaxation upon ultrashort laser excitation of ferrous alloys. Their role defining energy deposition and primary microscopic material response to the laser irradiation is outlined. Nonequilibrium thermodynamic properties of 316L stainless steel are determined from first-principles calculations. Taking into account the complexity of multi-metallic materials, the density functional theory is first applied to describe the electronic density of states of an alloy stainless steel matrix as a function of electronic heating. An increase of the localization degree of the charge density was found to be responsible for the modification of the electronic structure upon electronic heating, with consequences on chemical potential, electronic capacity and pressure. It is shown that the electronic temperature dependence of stainless steel thermodynamic properties are consistent with the behavior observed for pure $\gamma$-Fe, outlining the role of the main constituent in the same atomic arrangement. Assuming that similar behaviors extend to the transport properties, the transient electron-phonon coupling, optical properties and thermal conductivities of $\gamma$-Fe are derived based on density functional perturbation theory and ab initio molecular dynamics and extrapolated for steel. The insertion of accurate transport coefficients allows to improve current models and to achieve more realistic description of femtosecond pulse laser processing. Effects of fast temperature variation driving phase transitions and strong thermal stresses induced by the laser pulse are finally presented by combining first principle results to a nonequilibrium hydrodynamic approach.

\section{Introduction}

The development of material processing is largely driven by off-stoichiometric disordered materials, whereas their complex microscopic behavior under ultrafast laser irradiation remains mostly inaccessible with current theoretical approaches. Particularly, stainless steels constitute a specific kind of alloys containing a certain amount of chromium that via surface oxidation produces a dense passive layer conferring corrosion resistance to the material. This crucial property is responsible for a largescale use of stainless steels spreading into many industrial domains where both the tailorable properties of steels and corrosion resistance are required. Unsurprisingly, these materials are also of interest for technological applications related to laser manufacturing, especially as they 
have been found to respond favourably to ultrashort laser irradiation. ${ }^{1}$ For instance, they are used in laser process development and recently in nanostructuring with applications in tribology, ${ }^{2}$ wettability ${ }^{3}$ and optical functionalization ${ }^{4}$ fields. Requiring accurate design and control, these applications put forward the challenge of an accurate description of laser-matter interaction from optical, thermal and mechanical point of view. This remains difficult due to the lack of nonequilibrium thermodynamic properties evolving with the electronic excitation induced by ultrashort laser exposure.

A general laser-matter interaction scenario goes on the following lines. By focusing an intense energy beam into a small and confined volume of matter in solid state, ultrashort laser irradiations produce a large excitation of electrons within the band structure. ${ }^{5}$ Via electron-electron collisions, these excited electrons redistribute momentum and energy and reach a Fermi-Dirac distribution within tens of femtoseconds timescale. ${ }^{6}$ The electronic subsystem is thus rapidly thermalized and can be defined by an electronic temperature $T_{e}$ reaching thousands of Kelvin, while the lattice system remains mostly unaffected and characterized by a low temperature $T_{i}$. The increase of $T_{e}$ with respect to $T_{i}$ refers to the so-called electron-phonon nonequilibrium state. At larger timescales, up to few picoseconds, ${ }^{7}$ the energy stored in the electronic subsystem is transferred to the lattice by electron-phonon coupling. This progressively leads to the return of the equilibrium $\left(T_{e}=T_{i}\right)$ while the energy is further dissipated through the thermal diffusivity inside the material.

These temperature evolutions are modelled through the two-temperature model relying on numerous thermodynamic parameters, among them the electronic heat capacities, the electron-phonon coupling and the thermal conductivity, ${ }^{8,9}$ all of them subject of approximation or sources of uncertainties. The electronic pressure is also of importance as it plays a significantly role in hydrodynamic models. ${ }^{10}$ This model is based on the assumption that both ionic and electronic systems can be efficiently described by two separated and effective tem- peratures which is found to be consistent with experimental observations. ${ }^{11,12}$ The thermodynamic conditions experienced by the material under irradiations strongly depends on these nonequilibrium properties. However, experimental evaluation of these properties is difficult as excited solids in steady states cannot be created. ${ }^{13-15}$ This is the main advantages of first-principles methods, where a finite electronic temperature can be applied and properties derived accordingly. ${ }^{16,17}$

In the present study, we investigate the evolution of 316L stainless steel properties under ultrafast laser-induced electron-phonon nonequilibrium. From electronic structure calculations, the temperature dependent density of electronic states are obtained. They give access to electronic thermodynamic quantities, as chemical potentials, heat capacities, pressures and free electron numbers. This is followed by the evaluation of transient transport properties, with the calculation of the electron-phonon coupling, the optical indices and thermal conductivities. Finally, the thermodynamic and kinetic conditions leading to the transient phase transformation in a surface region of a stainless steel sample irradiated by an ultrashort laser pulse are discussed in light of the results of a nonequilibrium hydrodynamic simulation.

\section{Ab initio calculations details}

The calculations are carried out in the framework of the density functional theory $(\mathrm{DFT})^{18,19}$ extended to non-zero temperatures. ${ }^{20}$ The finite electronic temperature is taken into account directly and indirectly through the Fermi-Dirac distribution of electrons and by minimizing the free energy with respect to the electronic entropy term. ${ }^{21}$ The exchange and correlation part is treated by generalized gradient approximations (GGA) in the form parameterized by Perdew, Burke and Ernzerhof. ${ }^{22}$ The Abinit package is used, ${ }^{23}$ which is based on a plane-waves description of the electronic wave functions. In order to take into account nuclei and core electrons, projector augmented-waves (PAW) atomic data ${ }^{24-26}$ 
are used for the atoms constituting the 316L stainless steel. For $\gamma$-Fe a Troullier-Martins (TM) pseudo-potential ${ }^{27}$ is considered to simplify linear response function for the calculation of the electron phonon coupling constant. The Monkhorst-Pack mesh of the reciprocal space, ${ }^{28}$ as well as the cutoff energy of plane-waves have been designed depending on the size of the cell and the kind of calculations.

Off-stoichiometric disorder refers to materials having multiple constituents in solid solution on the material lattice or sub-lattices. In our calculations, we have used the concept of special quasirandom structures (SQS), which has emerged in recent times as a powerful approach for the calculation of total energy and related properties in disordered phase. ${ }^{29,30}$ Proposed by Zunger et al., ${ }^{31}$ this method is specially designed to order supercells with a limited number of atoms constructed in such a way that the most relevant pair and multi-site correlation functions closely mimic the ensemble-averaged correlation functions of the random substitutional alloy. Since the atomic distributions on lattice sites in an SQS are determined on the basis of matching correlation functions, it can accurately capture the local environments in random alloys. Austenitic steels crystallize within a face centered cubic structure (FCC), the solid solution is modelled within a 108 atoms supercell generated by the ATAT package. ${ }^{32}$ It corresponds to a $3 \times 3 \times 3$ conventional cell of FCC. 316L stainless steel has a constituent mass composition of : $65.9 \% \mathrm{Fe}, 16-18 \% \mathrm{Cr}, 10.5-13 \% \mathrm{Ni}$, $2-2.5 \%$ Mo, $2 \%$ Mn, $1 \%$ Si, $0.04 \%$ P, $0.03 \%$ S and $0.02 \% \mathrm{C}$. We first approximated this composition to the most abundant constituents, i.e. $\mathrm{Fe}, \mathrm{Cr}$ and $\mathrm{Ni}$, leading to the $\mathrm{Fe}_{73} \mathrm{Cr}_{21} \mathrm{Ni}_{14}$ supercell and corresponding to a mass composition of $68.1 \% \mathrm{Fe}, 18.2 \% \mathrm{Cr}, 13.7 \% \mathrm{Ni}$. A picture of the corresponding supercell is provided in Figure 1. Beforehand, we conducted a geometry optimization, including cell volume and atomic positions. The cubic shape of the cell was conserved to maintain the FCC arrangement. To reduce computational costs while keeping accuracy, we performed a set of tests that led to this minimalistic set of convergence parameters: a $k$-point grid of $3 \times 3 \times 3$ with

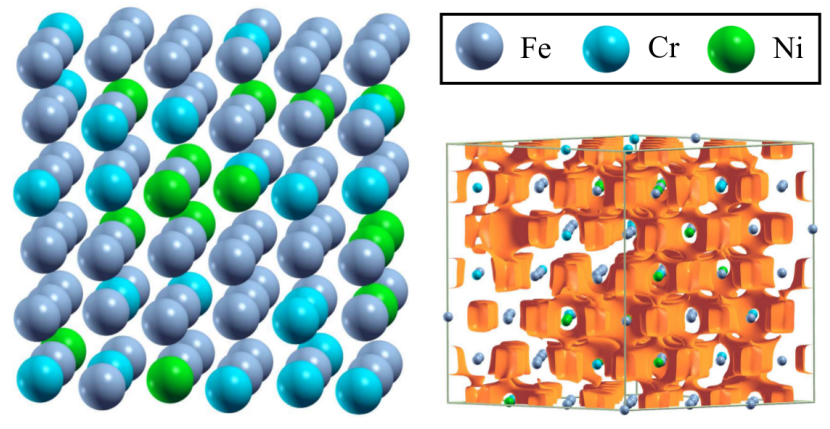

Figure 1: The 108 atoms supercell of 316L stainless steel generated with the SQS method (left), and a representation of the electronic density at $T_{e}=4 \times 10^{4} \mathrm{~K}$ (right).

a cutoff energy of $15 \mathrm{Ha}$ and a smearing parameter of $0.001 \mathrm{Ha}$. Once forces were below $5 \times 10^{-4} \mathrm{eV} / \AA$ the convergence was considered to be reached, leading to a supercell parameter of $10.47 \AA$, close to the experimental value of $10.77 \AA$. Further electronic temperature dependent calculations were performed with a $10 \times$ $10 \times 10 k$-point grid and with a similar cutoff energy.

To ensure the accuracy of PAW atomic data and TM pseudo-potentials, we first focused on the determination of key properties of the pure metals formed by the main constituents of the steel. Accordingly, we first considered Fe, Cr and $\mathrm{Ni}$ phases at ambient conditions and additionally the high temperature phase of Fe as it adopts similar crystal structure as austenitic steels. The electronic configurations considered are $3 d^{6} 4 s^{2}, 3 s^{2} 3 p^{6} 3 d^{5} 4 s^{1}$ and $3 d^{8} 4 s^{2}$ for $\mathrm{Fe}, \mathrm{Cr}$ and $\mathrm{Ni}$ respectively. In standard conditions, Fe crystallizes in a body cubic centered structure (BCC) that corresponds to the so-called $\alpha$ phase, it shows a ferromagnetic (FM) order. Ni adopts a FCC structure with a FM order, and Cr crystallizes in a BCC structure, exhibiting a complex antiferromagnetic (AFM) spin-density-wave ${ }^{35}$ as a ground state. Having a dominant composition of Fe atoms, austenitic stainless steel results are compared to those obtained on Fe with the FCC structure. It corresponds to a high temperature allotrope known as the $\gamma$-Fe phase characterized by a complex spin spiral magnetism leading to local FM orders and global AFM properties. ${ }^{36}$ As a precise 
Table 1: Theoretical and experimental values of lattice parameters $(\AA)$, bulk moduli (GPa) and magnetic moments per atom for the pure metals, as main constituents of the steel.

\begin{tabular}{cccccccc} 
Metal & Chem. Struc. & $\mathrm{a}^{\text {th }}$ & $\mathrm{a}^{\text {ref }}$ & $\mathrm{B}^{\text {th }}$ & $\mathrm{B}^{\text {ref }}$ & $\mu_{B}^{\text {th }}$ & $\mu_{B}^{\text {ref }}$ \\
\hline$\alpha-\mathrm{Fe}$ & $\mathrm{BCC}$ & 2.84 & $2.87^{a}$ & 175 & $170^{a}$ & 2.22 & $2.22^{a}$ \\
$\gamma-\mathrm{Fe}$ & $\mathrm{FCC}$ & 3.51 & {$[3.39-3.45]^{b}$} & 275 & {$[294-346]^{b}$} & - & - \\
$\mathrm{Cr}$ & $\mathrm{BCC}$ & 2.87 & $2.91^{a}$ & 171 & $160^{a}$ & 0.27 & - \\
$\mathrm{Ni}$ & $\mathrm{FCC}$ & 3.52 & $3.52^{a}$ & 188 & $180^{a}$ & 0.62 & $0.61^{a}$ \\
\hline
\end{tabular}

${ }^{a}$ Experimental data from Ref. $33 ;^{b}$ Theoretical data from Ref. 34.

determination of these magnetic properties is not the scope of this paper, the magnetic orders are simplified to FM orders for $\alpha$-Fe and $\mathrm{Ni}$, a classic AFM order for $\mathrm{Cr}$ and a non-magnetic order for $\gamma$-Fe. Calculations are performed with a $40 \times 40 \times 40$ or equivalent mesh of the reciprocal space and a cutoff energy of $40 \mathrm{Ha}$. Lattice parameters, bulk moduli and magnetic moments of these metal phases are provided in Table 1. Theoretical results show a good agreement with experimental data, confirming the reliability of the potentials used for the modelling of the 316L stainless steel.

\section{$T_{e}$ dependent Density of States and nonequilibrium thermodynamic properties}

Laser irradiation effects are first taken into account through the electronic temperatures, which constitute, to a first approximation, primary excitation effect. They are applied on the fixed and cold lattice previously optimized. The densities of electronic states (DOS) are computed at 7 different electronic temperatures, from $10^{-2}$ to $4 \times 10^{4} \mathrm{~K}$. The bottom of the valence band of the electronic structure computed at $10^{-2} \mathrm{~K}$ is arbitrarily shifted in order to start at $0 \mathrm{eV}$, then all $T_{e}$ dependent DOS are shifted from the same value. The $T_{e}$ dependent electronic structures of 316L austenitic stainless steel and $\gamma$-Fe are provided in the Figure 2. The valence DOS of these metals are constituted of two distinct zones. A continuous background consisting of low density of states having a shape of a roughly square root shape of the energy - corresponds to the energy dispersion of spatially delocalized bands as $s$ or $p$ bands. Between 4 and $12 \mathrm{eV}$ a part consisting of high density of electronic states corresponds to bands having a higher spatial localization degree, i.e. $3 d$ bands that we refer to as the $d$ block. The difference in behaviour between $3 d$ band and $4 s p$ bands mainly lies in the main quantum number, whose value controls the spatial extension of the orbital. Accordingly, $3 d$ orbitals are less spatially extended than $4 s p$ ones, leading to weaker overlaps and interactions, and finally to a lower energy dispersion from which emerges the $d$ block.

Considering 316L stainless steel and $\gamma$-Fe at $T_{e}=10^{-2} \mathrm{~K}$, the densities of states are quite similar (Figure 2a and b). Stainless steel shows a larger and smoother $d$ block than $\gamma$-Fe, due to multiple constituents that modify the nuclei field and induce a symmetry lowering that allows atomic displacements from the positions of high symmetry. The Fermi energy is located within the $d$ block in both cases, indicating an electronic filling around $2 / 3$. The $d$ block part is the most affected by the increase of $T_{e}$. In both materials it tends to lower the density of the $d$ blocks in conjunction with an increase of their width. A shift of the $d$ block toward higher energies also occurs with the increase of the electronic temperature. This is attributed to an increase of the electronic screening with $T_{e}$ that originates in an electron transfer from spatially delocalized $s p$ bands toward localized $d$ bands. ${ }^{17}$ This induces a stronger localization of the electronic density around nucleus and reduces the effective electron-ion potential. This phenomenon occurs when the electronic chemical potential $\mu\left(T_{e}\right)$ is located within a partially 


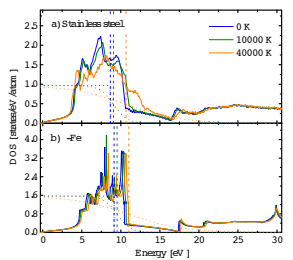

Figure 2: Electronic structures of 316L austenitic stainless steel (a) and $\gamma$-Fe (b) with the dependence on the electronic temperature. Blue, green and orange colors respectively correspond to $T_{e}=10^{-2}$, $10^{4}$ and $4 \times 10^{4} \mathrm{~K}$. In dotted curves, the Fermi Dirac distributions of electrons are provided with their characteristic electronic chemical potential in dashed vertical lines.

occupied $d$ block, as already noticed for $\mathrm{Ti}$ and W in Ref. 17.

Figure 3 shows thermodynamic properties of both 316L stainless steel and $\gamma$-Fe. We focus on the state parameters $\mu_{e}, C_{e}, P_{e}$ and $N_{e}$ with a dependency on the electronic temperature, as an approximate approach used to describe the conditions experienced by the material during the irradiation process. First the electronic chemical potential is provided in Figure 3a. This parameter is a signature of the electronic filling of the electronic structures. It is increasing with $T_{e}$, showing strong similarities between steel and $\gamma$-Fe. This increase with $T_{e}$ is first due to the balance of electronic states around the Fermi level with higher densities of filled electronic states at its left side and lower densities of empty electronic states at its right.
Depopulated electrons are thus accommodated by a displacement of the electronic potential toward higher energies. To a lower extent, $\mu\left(T_{e}\right)$ is also affected by the shift of the $d$ block toward higher energy with $T_{e}$. Evolution of the electronic chemical potential toward high energies results from two superimposed effects, asymmetry of occupied and unoccupied electronic states on one side, and shift of the $d$ block on the other side.

Being an indicator of the $T_{e}$ rise as a function of the deposited energy, the electronic heat capacity $C_{e}$ is obtained from the variation of the internal energy $E$ with respect to the electronic temperature as $C_{e}=\partial E / \partial T_{e}$. In magnitude, this thermodynamic quantity is similar for steel and Fe, as observable in Figure 3b. However, stainless steel rapidly shows an asymptotic behavior starting from $10^{4} \mathrm{~K}$ while a more complex change is observable for $\mathrm{Fe}$ at higher temperatures. The comparison of $C_{e}\left(T_{e}\right)$ values to the ones obtained for other pure metals ${ }^{37}$ confirms a quite linear relation of the electronic heat capacities with the number of valence electrons, as they respond collectively to the heating of the electronic subsystem.

With the increase of the electronic temperature, irradiated samples undergo an important increase of the electronic pressure $P_{e} \cdot{ }^{17}$ Both parameters may have important consequences in term of phase stability. ${ }^{38}$ The pressure is computed from the variation of the free energy $F$ with respect to the volume $V$ as $P_{e}=$ $-\partial F / \partial V=-\partial E / \partial V+T_{e} \partial S / \partial V$, with $S$ being the entropy of the system. The change of stainless steel electronic pressure upon excitation is provided in Figure 3c. It linearly increases up to $150 \mathrm{GPa}$ at $4 \times 10^{4} \mathrm{~K}$, a behavior which is again very similar to the one of iron within its FCC phase. Such important modifications of electronic conditions $\left(T_{e}\right.$ and $\left.P_{e}\right)$ might induce phases instabilities, especially since martensitic transformations are frequent for steels, ${ }^{39}$ but laser-induced diffusionless transformations are beyond the scope of this study.

While it has various meaning and definition depending on the applied field, ${ }^{17,37}$ the free electron number is a key parameter used in continuous nanoscopic optical and thermodynamic 


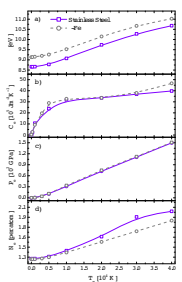

Figure 3: Evolution of a) electronic chemical potentials, b) electronic heat capacities, c) electronic pressures and d) number of free electrons as a function of the electronic temperature. Solid curves stand for stainless steel and dashed curves for $\gamma$ Fe.

models. Classically, it is evaluated according to the electronic configuration of isolated atoms ${ }^{40}$ and thus it disregards electronic transfers induced by solid state band structure as well as electronic temperature effects. According to this method, the free electrons are those belonging the most extended orbital of the electronic configuration i.e. the one having the highest main quantum number. Thus, free electron numbers from the classical approach are 2 for Fe, 1 for Cr and 2 for Ni. For our modelled steel $\mathrm{Fe}_{73} \mathrm{Cr}_{21} \mathrm{Ni}_{14}$, it leads to a number of free electrons per atom around of 1.8. To overcome restrictions induced by the classical approach, we developed an original and simple methodology based on density of electronic states. The main idea is that free electrons belong to spatially delocalized states that strongly interact, leading to a strong energy dispersion and to a DOS having a roughly square root shape of low den- sity (as Al). Accordingly, the $d$ block, that corresponds to spatially localized electronic states is manually removed and replaced by a square root function. The procedure is repeated for all $T_{e}$ dependent density of states and the resulting DOS is integrated with the unchanged FermiDirac function, leading to a number of free electrons evolving with the electronic temperature. ${ }^{17}$ These numbers are provided for $316 \mathrm{~L}$ stainless steel and $\gamma$-Fe in Figure 3d. At low electronic temperature, $N_{e}\left(T_{e}\right)=1.3$ electrons for both stainless steel and Fe. These values are significantly lower than expected from the classical approach, which originates in the capability of the band structure to allow the transfer of electrons between bands. With the rise of $T_{e}, N_{e}\left(T_{e}\right)$ increase for both materials, reaching about 2 free electrons at the highest temperature considered here.

\section{Transient transport proper- ties}

\section{Electron-phonon coupling}

The effective electron-phonon coupling controls the energy transfer from the electronic subsystem to the lattice. ${ }^{9}$ It is a crucial parameter giving access to relaxation times of the nonequilibrium state and describes the effectiveness of excitation conversion to heat. However, the derivation of this property requires extensive response linear calculations and is generally approximated. More specifically, the $T_{e}$ dependent effective electron phonon coupling $G\left(T_{e}\right)$ can be obtained from the knowledge of $\lambda\left\langle\omega^{2}\right\rangle$, associated to the density of electronic states $g:{ }^{41}$

$G\left(T_{e}\right)=\frac{\pi \hbar k_{B} \lambda\left\langle\omega^{2}\right\rangle}{g\left(\varepsilon_{F}, T_{e}\right)} \int_{-\infty}^{\infty}-g^{2}\left(\varepsilon, T_{e}\right)\left(\frac{\partial f}{\partial \varepsilon}\right) \mathrm{d} \varepsilon$,

where $\lambda$ is the electron phonon coupling constant, $\left\langle\omega^{2}\right\rangle$ is the second moment of the phonon spectrum. The latter is also related to the Debye temperature $\left\langle\omega^{2}\right\rangle \approx \theta_{D}^{2} / 2$. $\hbar$ and $k_{B}$ respectively correspond to the Planck and Boltzmann constants and $\varepsilon_{F}$ is the Fermi energy. The 
unknown parameters here, $\lambda$ and $\left\langle\omega^{2}\right\rangle$ can be obtained either from experimental approaches as thermoreflectivity measurements, ${ }^{42}$ or from electron-phonon coupling calculations. With a cell of 108 atoms, the theoretical modelling of electron-phonon interaction is out of calculation capabilities. Consequently, we used the electron resistivity experimental approach of Nath and Majumbar $^{43}$ to evaluate $\lambda\left\langle\omega^{2}\right\rangle$ :

$\lambda=\frac{\beta \hbar g\left(\varepsilon_{F}\right)\left\langle v_{F}\right\rangle^{2} e^{2}}{6 \pi k_{B}}$ and $\theta_{D}=\frac{h c_{s}}{2 k_{B}} \sqrt[3]{\frac{6 N}{\pi V}}$.

Here, $\beta$ is the stainless electronic resistivity of $\mathrm{Fe}_{66} \mathrm{Cr}_{20} \mathrm{Ni}_{14}$ the closest composition to our modelled stainless steel. $v_{F}$ is the Fermi velocity, $c_{s}$ is the effective speed of sound in the material, $\mathrm{N}$ and $\mathrm{V}$ respectively stand for the number of atoms and the volume of the cell. These equations give $\lambda=0.58$ and $\theta_{D}=492 \mathrm{~K}$, and are used to estimate $G\left(T_{e}\right)$ which is provided in Figure 4. From $T_{e}=10^{-2}$ to $8 \times 10^{3} \mathrm{~K}$, $G\left(T_{e}\right)$ exhibits high values, this is followed by a $50 \%$ decrease at intermediate temperatures, before it reaches a levelled behavior at high temperatures. This decrease is attributed to a $T_{e^{-}}$ induced Fermi broadening, bringing a significant amount of electrons into an energy range of lower density of states above 10-12 eV (see Figure 2a). This induces a decrease of the available transition space able to accommodate the energy transfers from electrons to phonons. To a lower extent, this behavior has already been observed for thermalized electrons for $\mathrm{Ni}^{6,41}$ Despite these significant evolutions with the increase of $T_{e}$, these values are very high compare to the most of pure metals, ${ }^{41}$ making the stainless steel a material that releases rapidly the energy of its electronic subsystem to the lattice. An other method provided by Petrov and Anisimov ${ }^{44}$ is available to assess the electronphonon coupling. Based on the electronic thermal conductivity and the low temperature electronic structure, one gets the value of 0.43 for $\lambda\left(\theta_{D}\right.$ unchanged $)$. This is $25 \%$ lower than in the previous evaluation, leading to an effective electron-phonon coupling $25 \%$ lower.

In order to compare these results with $\gamma$-Fe,

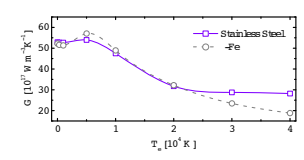

Figure 4: $T_{e}$ dependent effective electron-phonon coupling for stainless steel (solid line) and $\gamma$-Fe (dashed line). 
we computed the electron-phonon coupling constant from density functional perturbation theory. ${ }^{45} \lambda\left\langle\omega^{2}\right\rangle$ is defined as the second moment of the Eliashberg function $\alpha^{2} F(\Omega):{ }^{11}$

$$
\lambda\left\langle\omega^{2}\right\rangle=2 \int_{0}^{\infty} \omega \alpha^{2} F(\Omega) d \omega .
$$

A $q$-point grid of $4 \times 4 \times 4$ was used to obtain a converged Eliashberg spectral function, leading to an isotropic constant $\lambda$ equals to 0.62 and an associated Debye temperature of $458 \mathrm{~K}$. These values are very close to the experimental ones obtained for the steel. Accordingly, differences between stainless steel and $\gamma$-Fe $G\left(T_{e}\right)$ are weak, especially at low temperature. At high $T_{e}$, differences emerge and are related to the electronic structure evolution with $T_{e}$. For $\gamma$ $\mathrm{Fe}$, the electronic chemical potential leaving the $d$ block at high temperature leads to a significant decrease of the electron-phonon coupling and is not yet asymptotic despite the high $T_{e}$ considered here. This is a different behavior compared to stainless steel, where the $d$ block width increases with $T_{e}$, maintaining the electronic chemical potential in an energy region of high density of electronic states.

This different behavior originates from the various constituents of the stainless steel. Simple $\mathrm{Cr}, \mathrm{Fe}$ and $\mathrm{Ni}$ metals have $d$ block states of various density, with different energy location and electronic filling. Once added into a stainless steel, these initially proper characteristics are mixed and contributes to a larger $d$ block for steel than for $\gamma$-Fe (6.7 versus $5.8 \mathrm{eV}$ of width). Moreover, with the rise of $T_{e}$, the $d$ block width increases significantly more for steel than for $\gamma$-Fe, a phenomenon likely related to a stronger increase of the localization of the charge density for the steel. This is attributed to a lower electronic occupation of the $d$ block, since the composition percentage of $\mathrm{Cr}$ (6 valence electrons per atom) is greater than the one of $\mathrm{Ni}$ (10 valence electrons per atom) leading to a $13 \%$ deficit of electrons with respect to $\gamma$-Fe ( 8 valence electrons per atom). Additionally, the partial occupation of the $d$ block is lower for steel than for Fe, decreasing the pos- sibility for the electronic chemical potential to escape from the $d$ block. All these phenomena contribute to maintain $\mu\left(T_{e}\right)$ in a region of high density of states at high temperature leading to the difference between steel and Fe at high $T_{e}$.

\section{Optical properties and thermal conductivity}

In laser irradiation conditions, optical properties are crucial parameters as they characterize the material reflectivity and thus the amount and distribution of absorbed light energy. In addition, the optical properties determine the possibility of generating evanescent penetration of light below the surface. Optical coupling defines therefore the interaction with the possibility of large excursion of optical properties upon electronic heating. The thermal conductivity controls the diffusion of the thermal energy over lengths larger than the penetration depth, and thus is responsible for thermal energy confinement or spreading, with consequences on the cooling and matter state of the irradiated material. Both quantities are dependent on temperature and pressure conditions that are taken into account in a first-principles approach, proceeding in two steps. First an ab initio molecular dynamical simulation is performed in the desired conditions of ionic temperature and pressure. Once a pseudo-equilibrium is reached, ionic configurations are extracted and stand as being representative of the state of the matter in those conditions. Then, accurate DFT calculations are performed giving access to the corresponding condition-dependent electronic structures, that are used for the derivation of the properties of interest. Here, assuming that the similar behavior of $\gamma$-Fe and 316L stainless steel extends to their optical properties and thermal conductivities, and in the aim of decreasing computational costs, the optical properties are only computed for Fe, in its FCC phase.

The main point is to evaluate how optical properties evolve for Fe FCC at standard conditions, while its electronic temperature increases. It corresponds to the first step of the ulrashort irradiation prior any significant energy transfer from the electronic subsystem to 
the lattice. Accordingly, the molecular dynamic simulation is performed at $T_{i}=T_{e}=300 \mathrm{~K}$, in the framework of the isokinetic ensemble to keep the ionic temperature constant. A supercell of 16 iron atoms in a FCC lattice is used, with a reciprocal space meshed by a $k$-point grid equivalent to $3 \times 3 \times 3$. The modelling lasted 2 ps, from which an ionic configuration is extracted at the end of the run. Then, accurate electronic structure calculations are carried out involving a $k$-point grid equivalent to $10 \times$ $10 \times 10$ and a number of bands significantly increased to take into account the electronic temperatures. These calculations are coupled to the Kubo-Greenwood formalism for the determination of the optical properties, ${ }^{46}$ and to the Onsager coefficients giving access to the thermal conductivity. ${ }^{47}$ Here, optical properties and thermal conductivities are computed at $T_{i}=300 \mathrm{~K}$ and $T_{e}$ equals to $300,1.5 \times 10^{4}$ and $3 \times 10^{4} \mathrm{~K}$.

The corresponding $T_{e}$ dependent complex optical indices $\tilde{n}=n+i k$ are provided in Figure 5. The smooth evolution of the curves with the photon energy indicates a good convergence with respect to the mesh of the reciprocal space. Being a high temperature phase, optical properties of $\gamma$-Fe (FCC phase) are not available, consequently, we provided experimental data related to $\alpha$-Fe, the BCC ambient condition phase. Both electronic structures of these phases are comparable, with $d$ blocks having similar width and electronic occupation. The main difference lies in the presence of a pseudoband gap in the $d$ block for BCC while a more continuous density of states characterizes the one of FCC phase. This difference produces a dumping of optical properties for $\alpha$-Fe compared to $\gamma$-Fe, explaining the difference of optical properties between theoretical and experimental data. If we focus on theoretical data, the intraband component of $n$ and $k$ dominates in the photon energy range up to $1 \mathrm{eV}$. Since at $T_{e}=0 \mathrm{~K}$ the Fermi energy is located within the $d$ band, an interband contribution is also effective within the intraband part. The interband component extends up to 3 and $4 \mathrm{eV}$ for $n$ and $k$ respectively, corresponding to the energy width of the occupied $d$ block, an energy

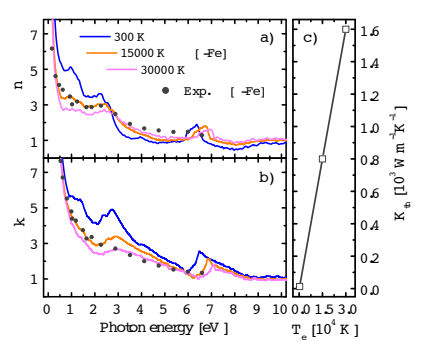

Figure 5: $T_{e}$ dependent optical indices of $\gamma$-Fe, real part in a) and imaginary part in b). The experimental data of $\alpha$-Fe is also provided in black dots. ${ }^{48,49}$ c) the thermal conductivity $\left(\mathrm{K}_{t h}\right)$, exhibiting a strong dependency on the electronic temperature.

domains that concentrates interband electronic transitions. The increase of $T_{e}$ produces an important redistribution of the electrons within the band structure as can be seen in Figure 2 . The corresponding Fermi broadening activates an increasing number of electronic transitions, whose effect is to dilute the interband signal, leading to a dumping of the interband component. Consequently, optical indices decrease in the photon energy range corresponding to the interband component as shown in Figure 5a and b. The $T_{e}$ dependent thermal conductivity is plotted in Figure 5c. A substantial increase of this quantity occurs with $T_{e}$, in agreement with the strong augmentation determined by Petrov et al. ${ }^{50}$ At low temperature, the computed value is $13 \mathrm{Wm}^{-1} \mathrm{~K}^{-1}$, close to the experimental value $\mathrm{K}_{t h}^{e x p}=15 \mathrm{Wm}^{-1} \mathrm{~K}^{-1},{ }^{51}$ it then increases to 800 and $1600 \mathrm{Wm}^{-1} \mathrm{~K}^{-1}$ at $1.5 \times 10^{4}$ and $3 \times 10^{4} \mathrm{~K}$, 
respectively. The significant modification of optical indices and the strong increase of the thermal conductivity with $T_{e}$ have important consequences on the energy absorption and transfer, and has to be taken into account for lasermatter interaction modelling as described in the next section.

\section{Two-temperature hydrody- namic simulation}

The hydrodynamic response of a stainless steel surface to ultrashort laser pulse heating has been investigated using the two-temperature hydrodynamics code Esther. Details of this code can be found elsewhere. ${ }^{10,52}$ Following laser excitation, the one-dimensional in-depth calculation of laser-matter interaction allows insight into the successive thermodynamic states for both electrons and ionic material. Thermomechanical excitation and subsequent relaxation stages define the energy distribution inside the material and are described accurately for laser fluence ranging from sub-ablation regime to warm dense plasma generation. To exploit the full range of electron properties calculated for temperatures up to $4 \mathrm{eV}$, the fluence has been set at $0.6 \mathrm{Jcm}^{-2}$, corresponding to 5 times the ablation threshold for a pulse duration of 100 fs (FWHM). We note there that the calculated threshold $\left(0.12 \mathrm{Jcm}^{-2}\right)$ is in good agreement with experimental values. ${ }^{53}$ The spatio-temporal dynamics of the irradiated surface is represented in Figure 6, where the evolution of electron and matter temperatures, density and total pressure in the expanding layers are given. Thermal excitation of the electrons constitutes the initial stage observed during laser absorption in the skin depth of the material as shown in Figure 6a.

Electronic properties determined by DFT method have been implemented to calculate the transient electronic heat capacity and pressure defining the thermodynamic state of the electron subsystem, as done in Refs. ${ }^{21,54,55}$ The thermodynamic and kinetic response of the metal is conditioned by the sharp electron temperature gradient but also by the strong electron-phonon coupling which leads to a fast energy transfer from the hot electrons to the lattice. This is reflected by the ionic temperature increase reaching a maximal value 1 ps after laser excitation as shown in Figure 6b. This short relaxation time is less than the hydrodynamic time scale $\tau_{h}=l_{T} / c_{s}=10 \mathrm{ps}$, where $l_{T} \simeq 45 \mathrm{~nm}$ is the thickness of the heated surface region while $c_{s}$ is the sound velocity. The characteristic size of the heated layer is mainly established by the competition of electron heat diffusion and electron-phonon relaxation and the dependence of the electron thermal conductivity with temperature as discussed in section 3 is crucial to determine the effective heat penetration depth. One can note that $l_{T}$ is particularly low for a metal, resulting to reduce the region of stress confinement. This relaxes by generating a high hydrodynamic momentum at the surface which can locally modify the surface topography. In that context, materials exhibiting a strong confinement of the energy have been found to foster the growth of laser-induced periodic surface structures. ${ }^{56}$ High contrast of these periodic pattern structures has been experimentally observed on stainless steel, which is likely related to the low extension of the heated layer.

At this fluence regime, the peak ionic temperature does not exceed the critical point, and material decomposition is supposed to take place through phase explosion. ${ }^{57-59}$ The dynamics of the ablated layers is clearly visible in Figure 6c depicting the kinetics of phase transformation through spatio-temporal density evolution. The solid-liquid interface is also shown by a dashed line showing that a thin liquid layer of $10 \mathrm{~nm}$ thickness was not expelled from the surface. Solidification process takes place 150 ps after irradiation and the ablation layer represents around $30 \mathrm{~nm}$ of material at $0.6 \mathrm{Jcm}^{-2}$. This calculated ablation depth is in excellent agreement with the experimental ones in similar ultrashort irradiation conditions. ${ }^{60,61}$ Turning to the investigation of the pressure evolution shown in Figure 6d, we predominantly observe the electronic contribution in the first picosecond which has not time to propagate before electron-ion equilibrium. A high pressure 


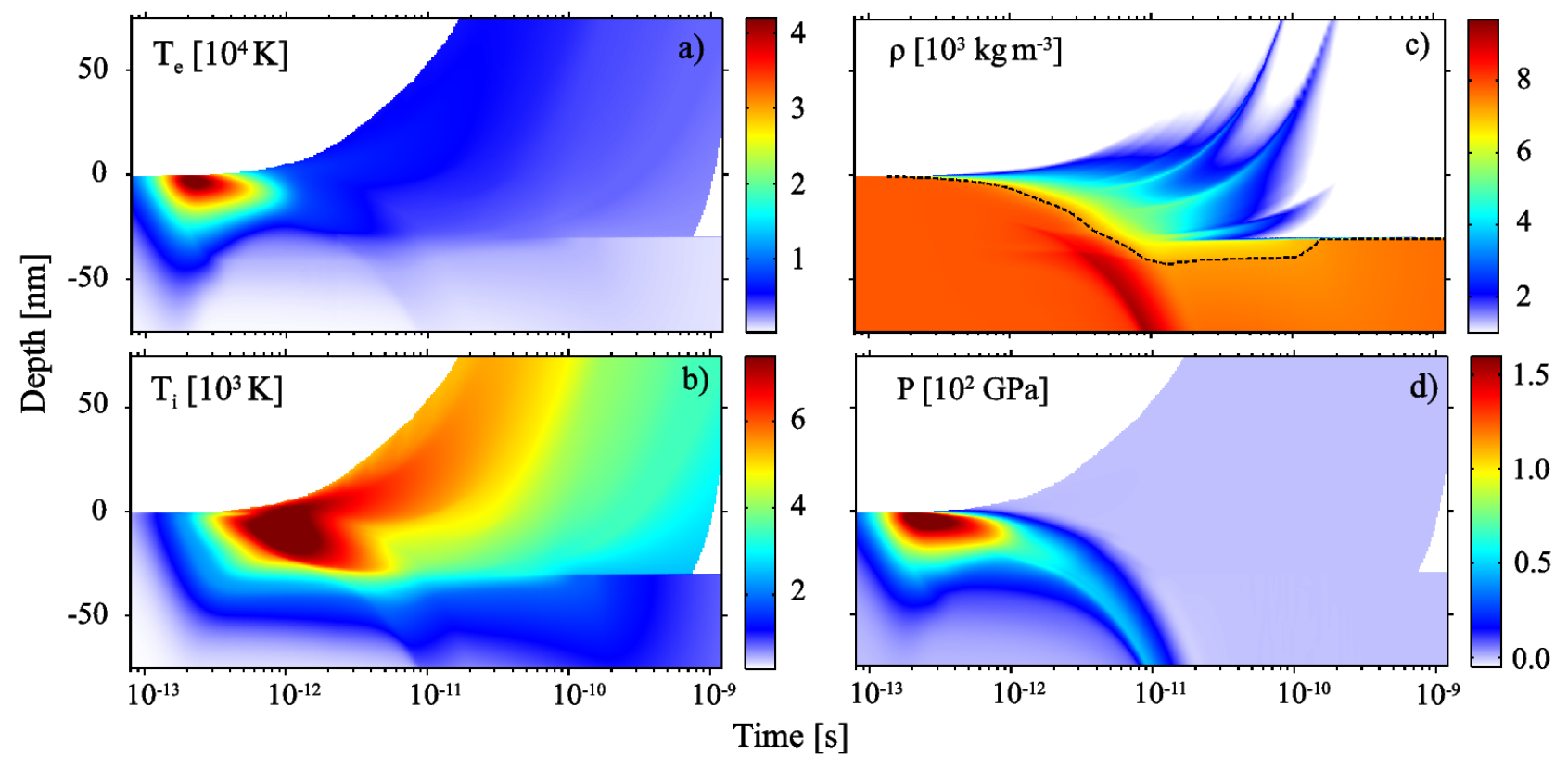

Figure 6: Spatio-temporal evolution of a) electronic temperature, b) matter temperature, c) material density and d) total pressure in a hydrodynamic simulation of a $316 \mathrm{~L}$ stainless steel target irradiated with a $100 \mathrm{fs}$ laser pulse at an incident fluence of $0.6 \mathrm{Jcm}^{-2}$. Simulation parameters integrate nonequilibrium electronic data presented in previous sections.

layer is formed near the surface, with a maximal pressure reaching $150 \mathrm{GPa}$ at $T_{e} \simeq 4 \times 10^{4} \mathrm{~K}$. Following the stage of energy transfer below the surface, an ionic wave propagates in-depth and toward the surface. The component reaching the free surface is reflected and transforms into a rarefaction wave resulting in tensile stresses (in white color in Figure 6d in the solid or liquid material, following the compression wave. ${ }^{54}$ These observations suggest that upon femtosecond irradiation relatively far from the melting threshold, extensive nonequilibrium DFT data are required to capture the complex phase transformation of the irradiated material.

\section{Conclusion}

In this study, the first instants of an ultrashort laser excited 316L stainless steel are modelled in the framework of the density functional theory. The thermal nonequilibrium properties have been calculated assuming a thermalization of the electronic subsystem and disregarding ionic temperature effects. The structure of the stainless steel has been rounded to its main constituents and generated according to the special quasirandom structure method in order to get a representative solid solution. Results have been compared to $\gamma-\mathrm{Fe}$, as it is the main constituent of 316L stainless steel, that has an austenite structure, i.e. a FCC atomic arrangement.

Within the electronic density of states, the $d$ block is found to enlarge and to shift toward higher energies due to the excitation process. This is in agreement with an increase of the electronic screening corresponding to a change of the electronic distribution from spatially delocalized states to more localized ones, as already observed for the pure metals $\mathrm{W}$ and $\mathrm{Ti}$. According to the unbalance of occupied electronic states and unoccupied ones from both side of the Fermi energy, the electronic chemical potential is found to shift toward higher energies to accommodate the excess of electrons for both metals.

The electronic heat capacities, electronic pressure, free electron numbers, effective electron-phonon coupling, and optical and thermal conductivities have been computed with a dependence on the electronic temperature. Electronic heat capacities are in agreement with the number of valence electrons per atom show- 
ing a clear asymptotic behavior for stainless steel. Electronic pressures increase significantly with $T_{e}$, as expected for intense laser irradiation conditions. However, at the difference of most of pure metals, the electron-phonon coupling is very high at low temperature, decreasing by a factor two at intermediate temperatures, and reaching an asymptotic behavior at high temperature. The interband signal for optical conductivities is weakened while the thermal conductivity significantly increases with the electronic temperature.

These nonequilibrium thermodynamic or transport properties are quite similar between $316 \mathrm{~L}$ stainless steel and $\gamma$-Fe, with some deviations at high temperature, especially concerning the number of free electrons and the electron-phonon coupling. This similar behavior tends to show that these results concerning both 316L stainless steels and $\gamma$-Fe can be extended to a set of similar austenitic steels. Accordingly, the dynamics of femtosecond laser ablation of a stainless steel target was studied based on a hydrodynamic simulation, relying on nonequilibrium DFT calculated data for electron temperature range commonly reached in materials processing.

Acknowledgement This work was supported by the ANR project DYLIPSS (ANR-12-IS04-0002-01) and by the LABEX MANUTECH-SISE (ANR-10-LABX-0075) of the Université de Lyon, within the program "Investissements d'Avenir" (ANR-11-IDEX0007) operated by the French National Research Agency (ANR). Part of the numerical calculations has been performed using resources from GENCI, project gen7041.

\section{References}

(1) Chichkov, B.; Momma, C.; Nolte, S.; von Alvensleben, F.; Tünnermann, A. Femtosecond, Picosecond and Nanosecond Laser Ablation of Solids. Appl. Phys. A 1996, 63, 109-115.

(2) Andersson, P.; Koskinen, J.; Varjus, S.; Gerbig, Y.; Haefke, H.; Georgiou, S.; Zh- mud, B.; Buss, W. Microlubrication Effect by Laser-Textured Steel Surfaces. Wear 2007, 262, 369-379.

(3) Wu, B.; Zhou, M.; Li, J.; Ye, X.; Li, G.; Cai, L. Superhydrophobic Surfaces Fabricated by Microstructuring of Stainless Steel Using a Femtosecond Laser. Appl. Surf. Sci. 2009, 256, $61-66$.

(4) Dusser, B.; Sagan, Z.; Soder, H.; Faure, N.; Colombier, J.-P.; Jourlin, M.; Audouard, E. Controlled Nanostructrures Formation by Ultra Fast Laser Pulses for Color Marking. Opt. Express 2010, 18, 2913-2924.

(5) Wellershoff, S.-S.; Hohlfeld, J.; Güdde, J.; Matthias, E. The Role of Electron-Phonon Coupling in Femtosecond Laser Damage of Metals. Appl. Phys. A 1999, 69, S99S107.

(6) Mueller, B. Y.; Rethfeld, B. Relaxation Dynamics in Laser-Excited Metals Under Nonequilibrium Conditions. Phys. Rev. B 2013, 87, 035139.

(7) Sun, C.-K.; Vallée, F.; Acioli, L.; Ippen, E. P.; Fujimoto, J. G. Femtosecond Investigation of Electron Thermalization in Gold. Phys. Rev. B 1993, 48, 1236512368 .

(8) Kaganov, M.; Lifshitz, I.; Tanatarov, L. Relaxation between Electrons and the Crystalline Lattice. Sov. Phys. Jetp-Ussr 1957, 4, 173-178.

(9) Anisimov, S.; Kapeliovich, B.; Perelman, T. Electron Emission From Metal Surfaces Exposed to Ultrashort Laser Pulses. Sov. Phys. Jetp-Ussr 1974, 39, $375-7$.

(10) Colombier, J.-P.; Combis, P.; Audouard, E.; Stoian, R. Guiding Heat in Laser Ablation of Metals on Ultrafast Timescales: An Adaptive Modeling Approach on Aluminum. New J. Phys. 2012, 14, 013039 . 
(11) Allen, P. B. Theory of Thermal Relaxation of Electrons in Metals. Phys. Rev. Lett. 1987, 59, 1460-1463.

(12) Cho, B. I.; Engelhorn, K.; Correa, A. A.; Ogitsu, T.; Weber, C. P.; Lee, H. J.; Feng, J.; Ni, P. A.; Ping, Y.; Nelson, A. J. et al. Electronic Structure of Warm Dense Copper Studied by Ultrafast X-Ray Absorption Spectroscopy. Phys. Rev. Lett. 2011, 106, 167601.

(13) Fann, W. S.; Storz, R.; Tom, H. W. K.; Bokor, J. Direct Measurement of Nonequilibrium Electron-Energy Distributions in Subpicosecond Laser-Heated Gold Films. Phys. Rev. Lett. 1992, 68, 2834-2837.

(14) Wang, X. Y.; Riffe, D. M.; Lee, Y.-S.; Downer, M. C. Time-Resolved ElectronTemperature Measurement in a Highly Excited Gold Target Using Femtosecond Thermionic Emission. Phys. Rev. B 1994, 50, 8016-8019.

(15) Ao, T.; Ping, Y.; Widmann, K.; Price, D. F.; Lee, E.; Tam, H.; Springer, P. T.; Ng, A. Optical Properties in Nonequilibrium Phase Transitions. Phys. Rev. Lett. 2006, 96, 055001.

(16) Recoules, V.; Clérouin, J.; Zérah, G.; Anglade, P. M.; Mazevet, S. Effect of Intense Laser Irradiation on the Lattice Stability of Semiconductors and Metals. Phys. Rev. Lett. 2006, 96, 055503.

(17) Bévillon, E.; Colombier, J. P.; Recoules, V.; Stoian, R. Free-Electron Properties of Metals Under Ultrafast Laser-Induced Electron-Phonon Nonequilibrium: A First-Principles Study. Phys. Rev. B 2014, 89, 115117.

(18) Hohenberg, P.; Kohn, W. Inhomogeneous Electron Gas. Phys. Rev. 1964, 136, B864-B871.

(19) Kohn, W.; Sham, L. J. Self-Consistent Equations Including Exchange and Correlation Effects. Phys. Rev. 1965, 140, A1133-A1138.
(20) Mermin, N. D. Thermal Properties of the Inhomogeneous Electron Gas. Phys. Rev. 1965, 137, A1441-A1443.

(21) Khakshouri, S.; Alfè, D.; Duffy, D. M. Development of an Electron-TemperatureDependent Interatomic Potential for Molecular Dynamics Simulation of Tungsten Under Electronic Excitation. Phys. Rev. B 2008, 78, 224304.

(22) Perdew, J. P.; Burke, K.; Ernzerhof, M. Generalized Gradient Approximation Made Simple. Phys. Rev. Lett. 1996, 7r, 3865-3868.

(23) Gonze, X.; Amadon, B.; Anglade, P.-M.; Beuken, J.-M.; Bottin, F.; Boulanger, P.; Bruneval, F.; Caliste, D.; Caracas, R.; Cote, M. et al. ABINIT: First-Principles Approach to Material and Nanosystem Properties. Comput. Phys. Commun. 2009, 180, 2582-2615.

(24) Blöchl, P. E. Projector Augmented-Wave Method. Phys. Rev. B 1994, 50, 1795317979 .

(25) Kresse, G.; Joubert, D. From Ultrasoft Pseudopotentials to the Projector Augmented-Wave Method. Phys. Rev. B 1999, 59, 1758-1775.

(26) Torrent, M.; Jollet, F.; Bottin, F.; Zérah, G.; Gonze, X. Implementation of the Projector Augmented-Wave Method in the ABINIT Code: Application to the Study of Iron Under Pressure. Comp. Mater. Sci. 2008, 42, 337-351.

(27) Troullier, N.; Martins, J. L. Efficient Pseudopotentials for Plane-Wave Calculations. Phys. Rev. B 1991, 43, 1993-2006.

(28) Monkhorst, H. J.; Pack, J. D. Special Points for Brillouin-Zone Integrations. Phys. Rev. B 1976, 13, 5188-5192.

(29) Grånäs, O.; Dutta, B.; Ghosh, S.; Sanyal, B. A New First Principles Approach to Calculate Phonon Spectra of Disordered Alloys. J. Phys.: Condens. Matter 2012, 24, 015402. 
(30) Steneteg, P.; Alling, B.; Abrikosov, I. A. Equation of State of Paramagnetic CrN From Ab Initio Molecular Dynamics. Phys. Rev. B 2012, 85, 144404.

(31) Zunger, A.; Wei, S.-H.; Ferreira, L. G.; Bernard, J. E. Special Quasirandom Structures. Phys. Rev. Lett. 1990, 65, 353-356.

(32) van de Walle, A.; Ceder, G. Automating First-Principles Phase Diagram Calculations. J. Phase Equilib. 2002, 23, 348-359.

(33) Kittel, C. Introduction to Solid State Physics, 8th ed.; John Wiley \& Sons, New York, 2004.

(34) Amador, C.; Lambrecht, W. R. L.; Segall, B. Application of Generalized Gradient-Corrected Density Functionals to Iron. Phys. Rev. B 1992, 46, 18701873 .

(35) Fawcett, E. Spin-Density-Wave Antiferromagnetism in Chromium. Rev. Mod. Phys. 1988, 60, 209-283.

(36) Mryasov, O. N.; Gubanov, V. A.; Liechtenstein, A. I. Spiral-Spin-Density-Wave States in FCC Iron: Linear-Muffin-TinOrbitals Band-Structure Approach. Phys. Rev. B 1992, 45, 12330-12336.

(37) Bévillon, E.; Colombier, J.; Recoules, V.; Stoian, R. First-Principles Calculations of Heat Capacities of Ultrafast Laser-Excited Electrons in Metals. Appl. Surf. Sci. 2015, 336, 79 - 84, E-MRS 2014 Spring Meeting. Symposium J. Laser Interaction with Advanced Materials: Fundamentals and Applications.

(38) Giret, Y.; Daraszewicz, S. L.; Duffy, D. M.; Shluger, A. L.; Tanimura, K. Nonthermal Solid-to-Solid Phase Transitions in Tungsten. Phys. Rev. B 2014, 90, 094103.

(39) Nishiyama, Z. Martensitic Transformation; Elsevier, 2012.
(40) Ashcroft, N. W.; Mermin, N. D. Solid State Physics; Holt, Rinehart and Winston, New York, 1976.

(41) Lin, Z.; Zhigilei, L. V.; Celli, V. ElectronPhonon Coupling and Electron Heat Capacity of Metals Under Conditions of Strong Electron-Phonon Nonequilibrium. Phys. Rev. B 2008, 77, 075133.

(42) Giri, A.; Gaskins, J. T.; Foley, B. M.; Cheaito, R.; Hopkins, P. E. Experimental Evidence of Excited Electron Number Density and Temperature Effects on Electron-Phonon Coupling in Gold Films. J. Appl. Phys. 2015, 117, 044305.

(43) Nath, T. K.; Majumdar, A. K. Resistivity Saturation in Substitutionally Disordered $\gamma-\mathrm{Fe}_{80-x} \mathrm{Ni}_{x} \mathrm{Cr}_{20}(14 \leqslant x \leqslant 30)$ alloys. Phys. Rev. B 1996, 53, 12148-12159.

(44) Petrov, Y. V.; Anisimov, S. Thermal Conductivity and Electron-Phonon Relaxation in a Metal Heated by a Subpicosecond Laser Pulse. J. Opt. Technol. 2006, 73, 368-370.

(45) Gonze, X. First-Principles Responses of Solids to Atomic Displacements and Homogeneous Electric Fields: Implementation of a Conjugate-Gradient Algorithm. Phys. Rev. B 1997, 55, 10337-10354.

(46) Mazevet, S.; Torrent, M.; Recoules, V.; Jollet, F. Calculations of the Transport Properties within the PAW Formalism. High Energy Density Phys. 2010, 6, 8488.

(47) Holst, B.; French, M.; Redmer, R. Electronic Transport Coefficients From Ab Initio Simulations and Application to Dense Liquid Hydrogen. Phys. Rev. B 2011, 83, 235120 .

(48) Johnson, P. B.; Christy, R. W. Optical Constants of Transition Metals: Ti, V, Cr, Mn, Fe, Co, Ni, and Pd. Phys. Rev. B 1974, 9, 5056-5070. 
(49) Ordal, M. A.; Bell, R. J.; Alexander Jr, R. W.; Newquist, L. A.; Querry, M. R. Optical Properties of Al, Fe, Ti, Ta, W, and Mo at Submillimeter Wavelengths. Appl. Opt. 1988, 27, 12031209 .

(50) Petrov, Y. V.; Inogamov, N.; Migdal, K. Thermal Conductivity and the ElectronIon Heat Transfer Coefficient in Condensed Media with a Strongly Excited Electron Subsystem. JETP Lett. 2013, 97, 20-27.

(51) Touloukian, Y. S.; Powell, R. W.; Ho, C.; Klemens, P. G. Thermophysical Properties of Matter; Y.S. Touloukian, IFI/Plenum, New York, 1970.

(52) Colombier, J. P.; Combis, P.; Bonneau, F.; Le Harzic, R.; Audouard, E. Hydrodynamic Simulations of Metal Ablation by Femtosecond Laser Irradiation. Phys. Rev. B 2005, 71, 165406.

(53) Pietroy, D.; Di Maio, Y.; Moine, B.; Baubeau, E.; Audouard, E. Femtosecond Laser Volume Ablation Rate and Threshold Measurements by Differential Weighing. Opt. Express 2012, 20, 29900-29908.

(54) Inogamov, N.; Petrov, Y. V.; Khokhlov, V.; Anisimov, S.; Zhakhovskiü, V.; Ashitkov, S.; Komarov, P.; Agranat, M.; Fortov, V.; Migdal, K. et al. The Effect of an Ultrashort Laser Pulse on Metals: TwoTemperature Relaxation, Foaming of the Melt, and Freezing of the Disintegrating Nanofoam. J. Opt. Technol. 2014, 81, 233-249.

(55) Wu, C.; Christensen, M. S.; Savolainen, J.-M.; Balling, P.; Zhigilei, L. V. Generation of Subsurface Voids and a Nanocrystalline Surface Layer in Femtosecond Laser Irradiation of a Single-Crystal Ag Target. Phys. Rev. B 2015, 91, 035413.
(56) Colombier, J.-P.; Garrelie, F.; Faure, N.; Reynaud, S.; Bounhalli, M.; Audouard, E.; Stoian, R.; Pigeon, F. Effects of Electron-Phonon Coupling and Electron Diffusion on Ripples Growth on Ultrafast-Laser-Irradiated Metals. $J$. Appl. Phys. 2012, 111, 024902.

(57) Cheng, C.; Xu, X. Mechanisms of Decomposition of Metal During Femtosecond Laser Ablation. Phys. Rev. B 2005, 72, 165415 .

(58) Lorazo, P.; Lewis, L. J.; Meunier, M. Thermodynamic Pathways to Melting, Ablation, and Solidification in Absorbing Solids Under Pulsed Laser Irradiation. Phys. Rev. B 2006, 73, 134108.

(59) Zhigilei, L. V.; Lin, Z.; Ivanov, D. S. Atomistic Modeling of Short Pulse Laser Ablation of Metals: Connections Between Melting, Spallation, and Phase Explosion. J. Phys. Chem. C 2009, 113, 1189211906.

(60) Mannion, P. T.; Magee, J.; Coyne, E.; O'Connor, G. M.; Glynn, T. J. The Effect of Damage Accumulation Behaviour on Ablation Thresholds and Damage Morphology in Ultrafast Laser MicroMachining of Common Metals in Air. Appl. Surf. Sci. 2004, 233, $275-287$.

(61) Le Harzic, R.; Breitling, D.; Weikert, M.; Sommer, S.; Föhl, C.; Valette, S.; Donnet, C.; Audouard, E.; Dausinger, F. Pulse Width and Energy Influence on Laser Micromachining of Metals in a Range of 100 fs to 5 ps. Appl. Surf. Sci. 2005, 249, 322 -331 . 


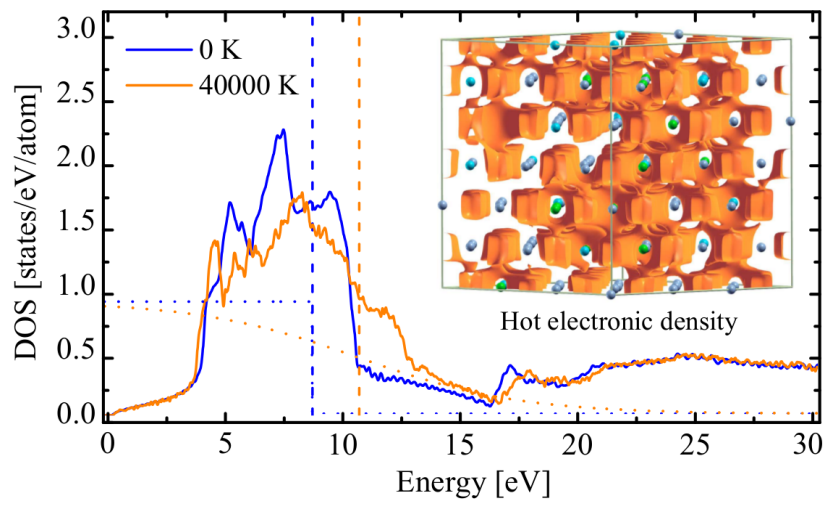

Table of contents graphic 